Pacific Journal of Mathematics

THE CONVERGENCE-PRESERVING REARRANGEMENTS OF 


\title{
THE CONVERGENCE-PRESERVING REARRANGEMENTS OF REAL INFINITE SERIES
}

\author{
Gerald S. Stoller
}

\begin{abstract}
Let $\mathscr{C}$ be the set of all permutations of the natural numbers that carry convergent real infinite series into convergent real infinite series. A strictly algebraic necessary and sufficient condition which determines $\mathscr{C}$ is given. $\mathscr{C}$ is seen to be a monoid but not a group. The maximum subgroup of $\mathscr{C}$ is shown not to be normal in $\mathscr{C}$.

A related set of permutations are those that preserve the sum of a convergent real infinite series when they carry that series to a convergent real series. This set of permutations is not a monoid. By exhibiting three different sufficient conditions for a permutation to belong to this set, we see that necessary and sufficient conditions determining this set will be difficult to ascertain.
\end{abstract}

Any automorphism of $\boldsymbol{N}$, the set of nonnegative integers, determines a unique rearrangement of infinite series. This correspondence is an anti-isomorphism. A subset of $S_{N}$, the group of all permutations of $N$, is defined by the condition that it carries convergent real infinite series into convergent real series. An algebraic condition can be shown to specify the same subset. We will see that this subset is a submonoid of $S_{N}$, but not a subgroup.

The problem of determining $\mathscr{C}$, the set of all permutations of $N$ that carry convergent real infinite series into convergent real infinite series, was considered nearly impossible to solve by E. Borel [2, page 101]. A necessary and sufficient condition on permutations of $\boldsymbol{N}$ for them to preserve convergence of real infinite series appears in [3, Prob. 5.2.2]. Letting $\sigma$ denote a permutation of $N$, this condition requires that there exists a finite cardinal $k$ such that $\sigma([0, n])$ can be expressed as the union of not more than $k$ intervals of $N$ for each $n \in N$. In fact, the set of permutations defined by this condition preserves convergence of infinite series (and their individual sums) in any normed abelian semigroup. Moreover, this is the largest set of permutations that preserve convergence in the family of all normed abelian semigroups. (For some normed abelian groups [e.g., the $p$-adics] the set of permutations preserving convergence is larger.)

$\mathscr{C}$ is easily shown to be a submonoid by using the convergence preservation property. We show that $\mathscr{C}$ is not a subgroup by establishing the necessary and sufficient conditions of [3, Prob. 5.2.2] and then exhibiting the permutation $\rho$ defined by 


$$
\begin{gathered}
(\forall n \in N)(\forall m \in[0, n]) \\
\rho(n(n+1)+2 m)=n(n+1)+m \text { and } \\
\rho(n(n+1)+2 m+1)=(n+1)^{2}+m .
\end{gathered}
$$

(We can also exhibit a convergent real infinite series that is carried into a divergent real infinite series by $\rho^{-1}$.)

Let $\mathscr{F} \mathscr{G}$ be the maximum subgroup of $\mathscr{C}$. We now focus on how $\mathscr{I} \mathscr{G}$ resides in $\mathscr{C}$.

THeOREM 1. $\mathscr{I} \mathscr{G}$ is not normal in $\mathscr{C}$.

Proof. Pick $\tau \in \mathscr{C}$ such that $N$ can be decomposed into intervals of ever increasing lengths on which $\tau$ acts as depicted in Fig. 1. Let $\sigma \in \mathscr{J} \mathscr{G}$ act on these intervals by cycling the left half interval into the right half interval, and vice versa (see Fig. 2). Note $\sigma^{2}=$ $e$, the identity permutation. $\tau \sigma \tau^{-1}$ acts on the left quarter of such an interval as shown in Fig. 3. Because of the increasing number of gaps, we have $\tau \sigma \tau^{-1} \notin \mathscr{C}$.

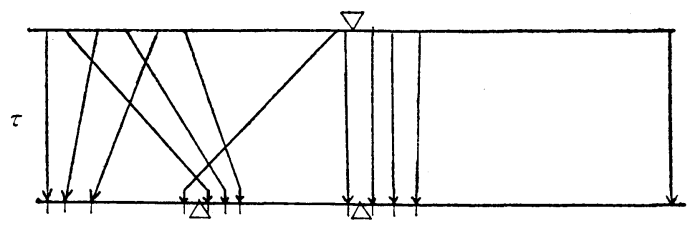

FIGURE 1

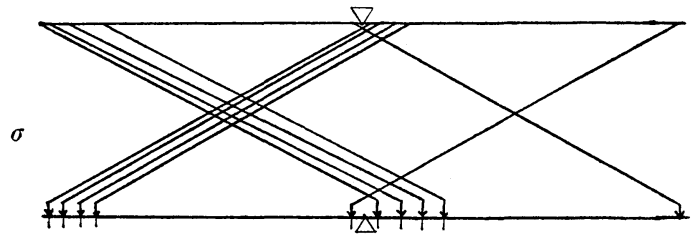

FIGURE 2

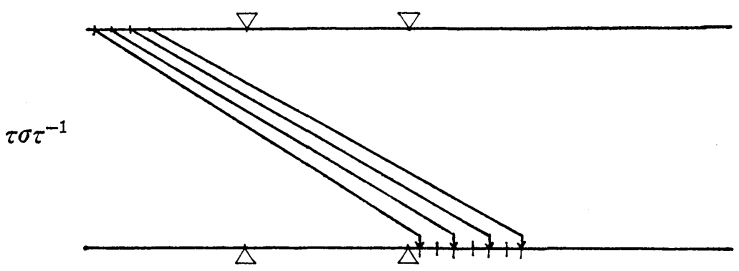

FiguRE 3

$\mathscr{I} \mathscr{G}$ contains all the torsion elements of $\mathscr{C}$. In fact, we also have $\tau \in \mathscr{C} \backslash \mathscr{I} \mathscr{G}$ implies $\tau^{n} \notin \mathscr{I} \mathscr{G}$ for all $n \in N \backslash\{0\}$. Since $\mathscr{C}$ is not a group, we have elements of period 0 in $\mathscr{C}$. $\mathscr{J} \mathscr{G}$ also contains elements of period 0 , for instance the element $\kappa$ of $S_{N}$ which cycles 
arbitrarily long sequences of nonnegative integers one position to the right and takes the last integer of the sequence into the first integer of the sequence.

The subgroup lattice of $\mathscr{J} \mathscr{G}$ appears to be rather rich, and many conditions can be used to pick out subgroups of $\mathscr{J} \mathscr{G}$. Three such conditions on $\sigma \in \mathscr{J} \mathscr{G}$ are:

(i ) $0<\inf \sigma(n) / n$ and $\sup \sigma(n) / n<\infty$.

(ii) $\lim \sigma(n) / n=1$.

(iii) $|\sigma(n)-n|$ is bounded.

Each condition implies the previous one. The last condition by itself defines a subgroup of $S_{N}$ that is a proper subgroup of $\mathscr{J} \mathscr{G}$. Subgroups of $S_{N}$ defined by these three conditions will be used in another paper.

A related set of permutations is composed of those permutations that, when they carry a convergent real infinite series into a convergent real infinite series, both series have the same sum (i.e., the permutation preserves sums of convergent real infinite series). This set is not closed under composition, as can be derived from the following theorem.

THEOREM 2. Every permutation in $S_{N}$ can be written as the product of two permutations, each of which maps $[0, n]$ onto $[0, n]$ for infinitely many $n \in N$.

Proof. Pick any $\rho \in S_{N}$. Pick any $m_{0} \in N$ and define $\sigma$ on $\left[0, m_{0}\right]$ by making it equal to $\rho$ there. Let $m_{1}>\sup \left(\sigma\left(\left[0, m_{0}\right]\right)\right)$ and define $\sigma$ on $\left(m_{0}, m_{1}\right]$ so that it maps $\left[0, m_{1}\right]$ onto itself. Now define $\sigma^{\prime}$ on $\left[0, m_{1}\right]$ so that $\sigma \sigma^{\prime}$ agrees with $\rho$ on this interval. Let

$$
m_{2}>\sup \left(\sigma^{\prime}\left(\left[0, m_{1}\right]\right)\right)
$$

and define $\sigma^{\prime}$ on $\left(m_{1}, m_{2}\right]$ so that it maps $\left[0, m_{2}\right]$ onto itself. Now define $\sigma$ on $\left(m_{1}, m_{2}\right]$ so that $\sigma \sigma^{\prime}$ agrees with $\rho$ on this interval. Continuing in this way, we eventually get $\sigma$ and $\sigma^{\prime}$ as concluded.

Obviously, the permutations $\sigma$ and $\sigma^{\prime}$ of the above theorem preserve the sums of convergent real infinite series. A sufficient condition for $\sigma \in S_{N}$ to preserve the sums of convergent real infinite series is that there exist finite cardinals $k^{\prime}$ and $k$ such that, for infinitely many $n \in N, \sigma$ maps a union of not more than $k^{\prime}$ intervals that contains $[0, n]$ onto a union of not more than $k$ intervals. The above $\sigma$ and $\sigma^{\prime}$ satisfy this condition with $k^{\prime}=1$. However, this condition is not necessary, as can be seen by constructing a permutation satisfying the following condition, which implies that the two 
infinite series involved have the same sum, provided both are convergent. Let $\zeta \in S_{N}$. Let there be sequences $M$ and $M^{\prime}$ and $M^{\prime \prime}$ such that $\lim M_{k}=\infty$ and $(\forall k \in N) M_{k}<M_{k}^{\prime}<M_{k}^{\prime \prime}$ and let $\mathscr{J}_{k}, \mathscr{J}_{k}^{\prime}$, and $\mathscr{F}_{k}^{\prime \prime}$ be the collections of maximal intervals not containing 0 of $\zeta\left(\left[0, M_{k}\right]\right)$, $\zeta\left(\left[0, M_{k}^{\prime}\right]\right)$, and $\zeta\left(\left[0, M_{k}^{\prime \prime}\right]\right)$ respectively. If card $\left(\mathscr{F}_{k} \cap \mathscr{F}_{k}^{\prime \prime}\right)$ is finitely bounded, and $(\forall k \in N) \mathscr{J}_{k}^{\prime}=\mathscr{J}_{k} \cup \mathscr{F}_{k}^{\prime \prime}$, then whenever $\zeta$ (via its induced mapping of infinite series) carries one convergent series to another, both must have the same sum. Obviously, this second sufficient condition covers different ground from the first condition. A third condition is obtained by changing two hypotheses of the second condition: change $M_{k}^{\prime \prime}$ to $M_{k+1}$ throughout (in effect adding the hypothesis $\left.M_{k}^{\prime \prime}=M_{k+1}\right)$, and replace "card $\left(\mathscr{I}_{k} \cap \mathscr{J}_{k}^{\prime \prime}\right)$ is finitely bounded" by "card $\left(\mathscr{F}_{k} \cap \mathscr{F}_{k}^{\prime \prime}\right) / \inf \left(\operatorname{card}\left(\mathscr{F}_{k}\right)\right.$, card $\left.\left(\mathscr{F}_{k}^{\prime \prime}\right)\right)$ is bounded away from 1." Because of this hodgepodge of sufficient conditions, it appears unlikely that a necessary and sufficient condition can be stated.

A result by Riemann [1, page 368] shows the existence of permutations that carry one conditionally convergent series to another, but do not preserve the sum. A specific example can be constructed as in [1, Exer. 12-18]. Together with Theorem 2, such an example shows that the set of permutations which preserve the sum when they carry one convergent series to another is not closed under composition (and so is not a monoid).

Several open questions remain. $\bigcap_{\rho \in \mathscr{B}} \rho \mathscr{C} \rho^{-1}$ is the maximum normal submonoid of $\mathscr{C}$. Is it a subgroup too? This intersection contains the subgroup of $S_{N}$ that consists of the finite permutations (those that move only finitely many elements of $N$ ). This subgroup is the maximum normal subgroup of $S_{N}$ (see [6] and [5]; related work appears in [4]). Is this intersection larger than the subgroup of finite permutations? Is there a characterization of this intersection's elements that provides an easy test to see if an element belongs to this intersection?

Similar questions (and others) can be raised for both $\mathscr{J} \mathscr{G}$ and the smallest subgroup of $S_{N}$ containing $\mathscr{C}$. Of course, the first problem is to determine this subgroup, $\mathbf{U}_{j=1}^{\infty}\left(\mathscr{C}^{-1} \mathscr{C}\right)^{j}$. Is it proper?

\section{REFERENCES}

1. Tom M. Apostol, Mathematical Analysis, Addison-Wesley Publishing Co., Inc., Reading, MA, 1957.

2. E. Borel, Sur le changement de l'ordre des termes d'une serie semiconvergente, Bull. des Sciences Mathematique, (2) 14 (1890), 97-102.

3. J. A. Dieudonne, Foundations of Modern Analysis, Academic Press, New York, 1969. 4. Clement F. Kent, Constructive analogues of the group of permutations of the natural numbers, Trans. Amer. Math. Soc., 104 (1962), 347-362.

5. L. Onofri, Teoria delle sostituzioni che operano su una infinità numerabile di elementi, Ann. Mat. Ser. (4) 4 (1927), 73-106; 5 (1928), 147-168; 7 (1930), 103-130. 
6. J. Schreier and S. Ulam, Über die Permutationsgruppe der natürlichen Zahlenfolge, Studia Math., 4 (1933), 134-141.

7. Gerald S. Stoller, Example of a proper subgroup of $S_{\infty}$ which has a set-transitivity property, Bull. Amer. Math. Soc., 69 (1963), 220-221.

Received June 1, 1976 and in revised form January 5, 1977.

Polytechnic Institute of New York

333 JAY STREET

BROOKLYN, NY 11201

AND

Emi Medical Inc.

3555 WOODHEAD DRIVE

NORTHBROOK, IL 60062 



\section{PACIFIC JOURNAL OF MATHEMATICS}

EDITORS

RICHARD ARENS (Managing Editor)

University of California

Los Angeles, California 90024

C. W. Curtis

University of Oregon

Eugene, OR 97403

C. C. MOORE

J. DUGUNDJI

Department of Mathematics University of Southern Californa Los Angeles, California 90007

R. Finn AND J. Milgram Stanford University Stanford, California 94305

University of California

Berkeley, CA 94720

\section{ASSOCIATE EDITORS}

E. F. BeCKENBACH

B. H. NeUMANN

F. WOLF

K. YosHida

\section{SUPPORTING INSTITUTIONS}

UNIVERSITY OF BRITISH COLUMBIA UNIVERSITY OF SOUTHERN CALIFORNIA CALIFORNIA INSTITUTE OF TECHNOLOGY UNIVERSITY OF CALIFORNIA MONTANA STATE UNIVERSITY UNIVERSITY OF NEVADA, RENO STANFORD UNIVERSITY UNIVERSITY OF TOKYO UNIVERSITY OF UTAH NEW MEXICO STATE UNIVERSITY WASHINGTON STATE UNIVERSITY OREGON STATE UNIVERSITY UNIVERSITY OF WASHINGTON UNIVERSITY OF OREGON OSAKA UNIVERSITY 


\section{Pacific Journal of Mathematics \\ Vol. 73, No. 1 \\ March, 1977}

Thomas Robert Berger, Hall-Higman type theorems. $V \ldots \ldots \ldots \ldots \ldots \ldots \ldots$

Frank Peter Anthony Cass and Billy E. Rhoades, Mercerian theorems via

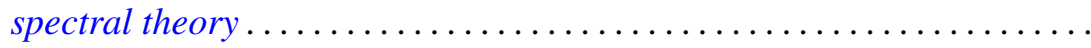

Morris Leroy Eaton and Michael David Perlman, Generating $\mathrm{O}(n)$ with

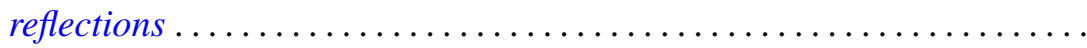

Frank John Forelli, Jr., A necessary condition on the extreme points of a

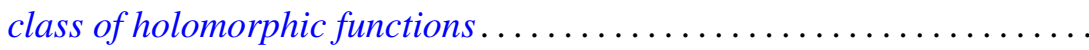

Melvin F. Janowitz, Complemented congruences on complemented

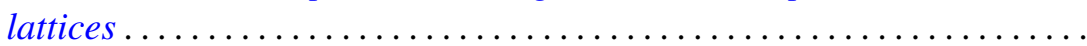

Maria M. Klawe, Semidirect product of semigroups in relation to amenability, cancellation properties, and strong $F \phi$ lner conditions....

Theodore Willis Laetsch, Normal cones, barrier cones, and the "spherical image" of convex surfaces in locally convex spaces ................

Chao-Chu Liang, Involutions fixing codimension two knots.............

Joyce Longman, On generalizations of alternative algebras .............

Giancarlo Mauceri, Square integrable representations and the Fourier

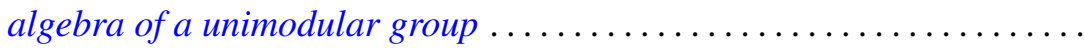

J. Marshall Osborn, Lie algebras with descending chain condition...

John Robert Quine, Jr., Tangent winding numbers and branched

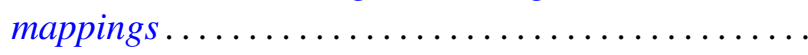

Louis Jackson Ratliff, Jr. and David Eugene Rush, Notes on ideal covers and associated primes .

H. B. Reiter and N. Stavrakas, On the compactness of the hyperspace of faces.

Walter Roth, A general Rudin-Carlson theorem in Banach-spaces ..

Mark Andrew Smith, Products of Banach spaces that are uniformly rotund in every direction.

Roger R. Smith, The R-Borel structure on a Choquet simplex ...

Gerald Stoller, The convergence-preserving rearrangements of real infinite series. ...

Graham H. Toomer, Generalized homotopy excision theorems modulo a Serre class of nilpotent groups...

Norris Freeman Weaver, Dehn's construction and the Poincaré conjecture....

Steven Howard Weintraub, Topological realization of equivariant intersection forms... 